\title{
Quantitative determination of optical transmission through subwavelength slit arrays in Ag films: Role of surface wave interference and local coupling between adjacent slits
}

\author{
D. Pacifici, H. J. Lezec, ${ }^{*}$ and Harry A. Atwater \\ Thomas J. Watson Laboratories of Applied Physics, California Institute of Technology, Pasadena, California 91125, USA \\ J. Weiner ${ }^{\dagger}$ \\ IRSAMC/LCAR, Université Paul Sabatier, 118 route de Narbonne, 31062 Toulouse, France \\ and IFSC/CePOF, Universidade de São Paulo, Avenida Trabalhador São-Carlense, 400-CEP 13566-590 São Carlos, São Paulo, Brazil
}

(Received 11 February 2008; published 7 March 2008)

\begin{abstract}
Measurement of the transmitted intensity from a coherent monomode light source through a series of subwavelength slit arrays in Ag films, with varying array pitch and number of slits, demonstrates enhancement (suppression) by factors of as much as 6 (9) when normalized to the transmission efficiency of an isolated slit. Pronounced minima in the transmitted intensity are observed at array pitches corresponding to $\lambda_{\mathrm{SPP}}, 2 \lambda_{\mathrm{SPP}}$, and $3 \lambda_{\mathrm{SPP}}$, where $\lambda_{\mathrm{SPP}}$ is the wavelength of the surface plasmon polariton (SPP). The position of these minima arises from destructive interference between incident propagating waves and pi-phase-shifted SPP waves. Increasing the number of slits to four or more does not increase appreciably the per-slit transmission intensity. A simple interference model fits well the measured transmitted intensity profile.
\end{abstract}

DOI: 10.1103/PhysRevB.77.115411

PACS number(s): 42.25.Fx, 73.20.Mf, 78.67.-n

\section{INTRODUCTION}

Since the first experimental report of "extraordinary optical transmission" through subwavelength hole arrays, ${ }^{1}$ considerable theoretical effort has been devoted to interpreting the essential physics of the process in both hole ${ }^{2-7}$ and slit $^{8-13}$ arrays. Roughly speaking, two points of view have emerged. Proponents of the first school ${ }^{2,3,8,14}$ have interpreted the transmission spectrum as excitation of the delocalized surface plasmon Bloch modes and identified transmission maxima with resonant excitation of these modes at wavelengths equal to integer multiples of the array pitch. The second school ${ }^{11-13,15,16}$ has emphasized interference between incident and surface waves and localized coupling between adjacent structures. Adherents of this approach predict transmission minima at the same positions where the Bloch mode excitation predicts maxima.

Experimental studies subsequent to the initial report ${ }^{1}$ demonstrated a number of unexpected features. Spectral transmission measurements ${ }^{17}$ revealed that, normalized to transmission of a single aperture, suppression, as well as enhancement, was a characteristic property of hole and slit arrays. Interferometric studies ${ }^{18-20}$ showed that the contribution of transient diffracted surface modes is as important as the surface plasmon polariton (SPP) guided mode in the immediate vicinity of the subwavelength object. The experimental setups of Refs. 1 and 17 consisted of an incoherent, broad-band light source dispersed through a scanning spectrophotometer and focused on fixed-period subwavelength hole and slit arrays. Transmitted intensity was detected in the far field as a function of the scanned wavelength. In that work, the spectral resolution and coherence length of light incident on the arrays therefore depended on instrumental parameters, and these, in turn, can affect the position and shape of the measured spectral features. Furthermore, the frequency dependence of the dielectric constant of $\mathrm{Ag}$ and other real metals is non-negligible in the range of typical wavelength scans from 450 to $900 \mathrm{~nm}$.

\section{EXPERIMENT}

In order to test the predictions of the two interpretive schools and to remove measurement ambiguities, we have undertaken a series of high-resolution measurements of the transmission through a series of slit arrays in which the spectral source is coherent, monomode, and at fixed frequency. Rather than scan the light source wavelength, we increment in $5 \mathrm{~nm}$ steps the array pitch of a series of slit arrays. The transmission measurement setup consists of a $\lambda_{0}=514.5 \mathrm{~nm}$, $5 \mathrm{~mW}, \mathrm{TEM}_{00}$ light beam from an Ar ion laser aligned to the optical axis of an inverted microscope. The beam is focused at normal incidence onto the sample surface through the microscope condenser and polarized TM (magnetic $H$-field component parallel to the long axis of the slits). Light intensity transmitted through each slit array is then gathered by a $50 \times$ microscope objective with a numerical aperture of 0.45 and detected with a liquid-nitrogen-cooled, charged-coupled device (CCD) array detector. Light intensity is obtained by integrating the signal over the entire region of interest in the CCD image and subtracting the background originating from electronic noise. Per-slit transmission intensities are obtained by correcting the transmitted intensity for the calculated collection efficiency of the microscope objective lens and normalizing the transmitted intensity for each series of gratings to the intensity collected from a single-slit structure. The series of slit arrays were milled with a focused-ion beam ( $\mathrm{Ga}^{+}$ions, $30 \mathrm{keV}$ ) in a $200 \mathrm{~nm}$ thick layer of silver evaporated onto a flat fused-silica microscope slide. The layout of slit-array structures consisted of a matrix of 9 rows and 140 columns. Each row was indexed by the slit number $N$ in the array and varied from $N=1$ to 9 . Each column was indexed by the array pitch $p$ starting from the first column at $p$ $=150 \mathrm{~nm}$ and incremented by $5 \mathrm{~nm}$ with each successive column. Thus, the pitch varied over a range from $p$ $=150$ to $845 \mathrm{~nm}$, from less than $\lambda_{\mathrm{SPP}}$ to greater than $2 \lambda_{\mathrm{SPP}}$. In subsequent measurements, the array pitch was extended to 

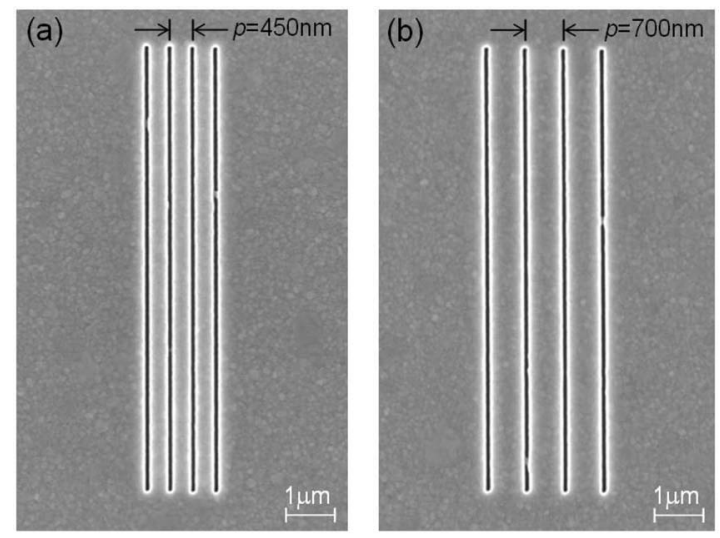

FIG. 1. Two typical elements in the overall structure layout. Panel (a) shows $N=4, p=450 \mathrm{~nm}$. Panel (b) shows $N=4, p$ $=700 \mathrm{~nm}$. Each slit is focused-ion beam milled through a $200 \mathrm{~nm}$ thick silver layer. Dimensions of each slit are $50 \mathrm{~nm}$ wide and $10 \mu \mathrm{m}$ long.

$3 \lambda_{\text {SPp. }}$ Each slit was milled $50 \mathrm{~nm}$ wide, $200 \mathrm{~nm}$ deep, and $10 \mu \mathrm{m}$ long, as shown in Fig. 1. The structured silver layer was covered by a second microscope slide, optically contacted to the silver surface by index-matching fluid ( $n$ $=1.46)$ so that the index change at the dielectric-silver interface was identical at both the input (incident) and output (transmitted) planes. The transmitted intensity of each successive array along a given row was recorded in the far field by the CCD as the sample was stepped using an $X-Y$ translation stage. The results are summarized in Fig. 2. Taking into account the collection efficiency of the microscope objective and the far-field angular distribution of the slit grating diffraction modes, we define $\eta=\left|H_{N}\right|^{2} /\left|H_{1}\right|^{2}$ as the ratio of

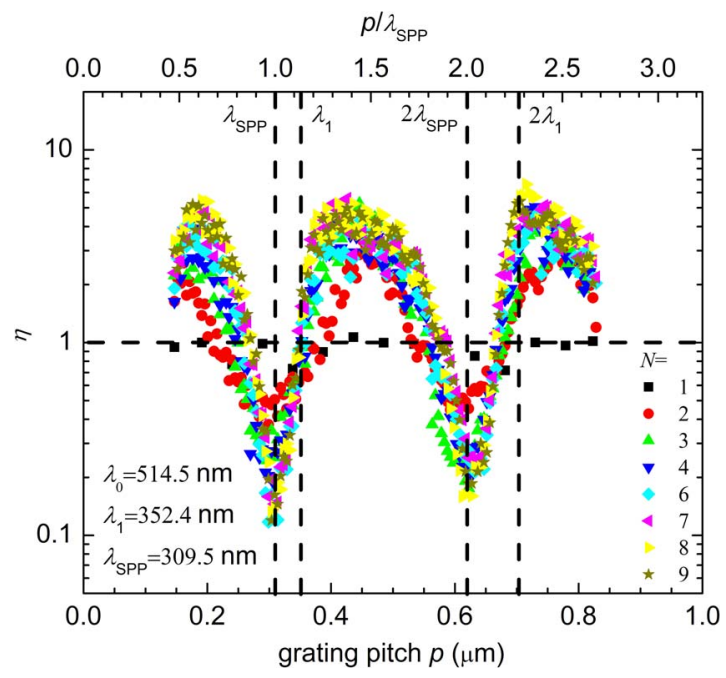

FIG. 2. (Color online) Normalized transmission intensity $\eta$ vs grating pitch (in micrometers on the lower abscissa and normalized to $\lambda_{\mathrm{SPP}}$ on the upper abscissa) for a series of slit arrays $N=1-9$. Gratings with $N=5$ were omitted due to defective fabrication. The wavelengths $\lambda_{0}, \lambda_{1}$, and $\lambda_{\text {SPP }}$ are, respectively, the free-space wavelength, the wavelength in fused silica $(n=1.46)$, and the wavelength of the surface plasmon polariton.

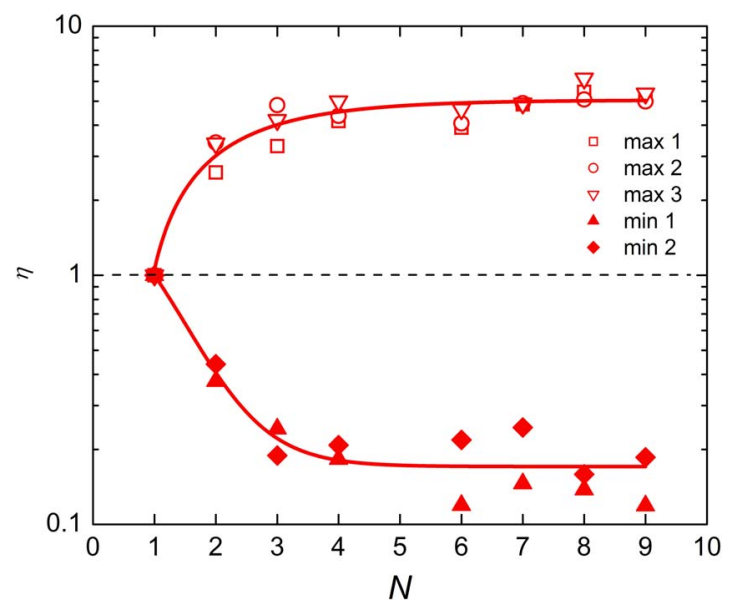

FIG. 3. (Color online) Normalized per-slit transmission intensity maxima and minima vs the number of slit elements in array $N$. The labels $1-3$ refer to the transmission intensity maxima and minima from left to right shown in Fig. 2. The solid lines are guides for the eyes.

the magnetic field intensity at the output aperture of each slit in an array of $N$ slits to the magnetic field intensity at the output aperture of an isolated slit.

\section{EXPERIMENTAL RESULTS}

Figure 2 plots $\eta$ vs array pitch for $N=1-9$ (except $N=5$, omitted due to defective fabrication). The results show that the transmission intensity for all arrays exhibits very similar behavior with transmission dropping to a minimum of $\simeq 0.1 \eta$ at an array pitch equal to $\lambda_{\mathrm{SPP}}$, then rising to a broad maximum of $\simeq 6 \eta$ before repeating similar behavior around $2 \lambda_{\text {SPP. }}$. The position of the minima is in accord with earlier predictions $^{11,12}$ and simulations ${ }^{13,21}$ and at variance with theory ${ }^{2,3}$ predicting transmission maxima at $\lambda_{\mathrm{SPP}}$.

The wavelength $\lambda_{\text {SPP }}$ was calculated from the usual formula for the guided wave on a flat,

$$
n_{\mathrm{SPP}}=\sqrt{\frac{\epsilon_{m} \epsilon_{d}}{\epsilon_{m}+\epsilon_{d}}},
$$

metal surface, ${ }^{22}$

$$
\lambda_{\mathrm{SPP}}=\frac{\lambda_{0}}{n_{\mathrm{SPP}}},
$$

where $\lambda_{0}$ is the incident wavelength, $\epsilon_{m}$ and $\epsilon_{d}$ are the dielectric constants of the metal and adjacent dielectric, respectively, and $n_{\mathrm{SPP}}$ is the effective surface index of refraction. In the present experiments, the dielectric constant of the structured silver sample was measured directly by ellipsometry at $\lambda_{0}=514.5 \mathrm{~nm}$ and determined to be $\epsilon_{m}=-9.3+0.18 i$. The dielectric constant of the fused-silica substrate is $\epsilon_{d}=+2.13$, and therefore $\lambda_{\mathrm{SPP}}=309 \pm 1 \mathrm{~nm}$.

Figure 3 plots the maximum and minimum values of $\eta$ for each of the $N$ grating series. Enhancement above single-slit transmission up to a factor of $\sim 6$ is observed as $N$ increases up to $N=4$. Above $N=3$, adding additional grating elements 
to the array does not significantly enhance the transmission. Similar behavior is observed for the transmission minima.

\section{DISCUSSION OF RESULTS}

These measurements support the view that transmission enhancement is dominated by nearest-neighbor slit scattering with the strength of the local interaction effectively screening contributions from more distant array elements. If Bloch surface modes, delocalized over the full extent of the array, played a dominant role, one would expect the per-slit intensity to increase with the number of elements in the array. The imaginary part of $k_{\mathrm{SPP}}$ determines the propagation distance along the surface limited by absorptive loss in the silver film. ${ }^{22}$ The estimated propagation distance is $8.5 \mu \mathrm{m}$, and therefore absorptive losses are negligible over the spatial extent of the grating structures.

Since the positions of transmission suppression and enhancement as a function of period are essentially independent of $N$, we can analyze the mechanism responsible for modulation by concentrating on the simplest case $N=2$. The normalized per-slit transmission intensity $\eta$ of an array of slit pairs with varying pitch $p$ is shown in Fig. 4(b). The intensity $\eta$ is plotted on a linear scale as a function of $p$ for devices milled into a Ag film of thickness $t=300 \mathrm{~nm}$ (hollow circles) in addition to the Ag film of thickness $t=200 \mathrm{~nm}$ described earlier (solid circles, replotted from Fig. 3). Comparison of the two data sets shows that the $\eta(p)$ modulation varies little with $t$ and is therefore essentially governed by the interaction between the two slits mediated by surface waves running along both facets of the structured metal film. Periodic minima are measured at slit-slit distances corresponding to integer multiples of $\lambda_{\mathrm{SPP}}\left(p=n \lambda_{\mathrm{SPP}}, n=1,2, \ldots\right)$. This observation is consistent with recent theoretical predictions for a two-slit system ${ }^{15}$ (albeit for a structure with only one SPPsustaining surface).

\section{A. Transmission model}

We have developed a simple model for $\eta(p)$. Figure 4(a) shows the essential idea. $H_{0}$ designates the $H$-field amplitude of the incident wave and $\beta H_{0}$ the amplitude component diffracted into the surface plasmon polariton mode at slit 1 . This mode with $\left|k_{\text {SPP }}\right|=2 \pi / \lambda_{\text {SPP }}$ propagates to the right along the surface until it reaches slit 2 . The phase accumulated by the surface wave at slit 2 is $e^{i k_{\mathrm{SPP}} p}$, where $p$ is the distance between the two slits. At slit 2, the SPP reconverts to a propagating mode with efficiency $\beta^{\prime}$ and interferes with the incident field. An identical process with the roles of slits 1 and 2 interchanged takes place at slit 1 . The superposition fields at the entrance side of slits 1 and $2, H_{0}+\beta \beta^{\prime} H_{0} e^{i k_{\mathrm{SPP}} p}$, are transmitted to the exit side with some overall efficiency $T$. A similar process takes place on the exit side of the film. Counterpropagating surface waves are again launched by diffractive scattering at the slit exits and interfered with the directly propagating mode at the opposite slit exit location. The total $H$-field amplitude at the output aperture of each slit is then given by the following expression
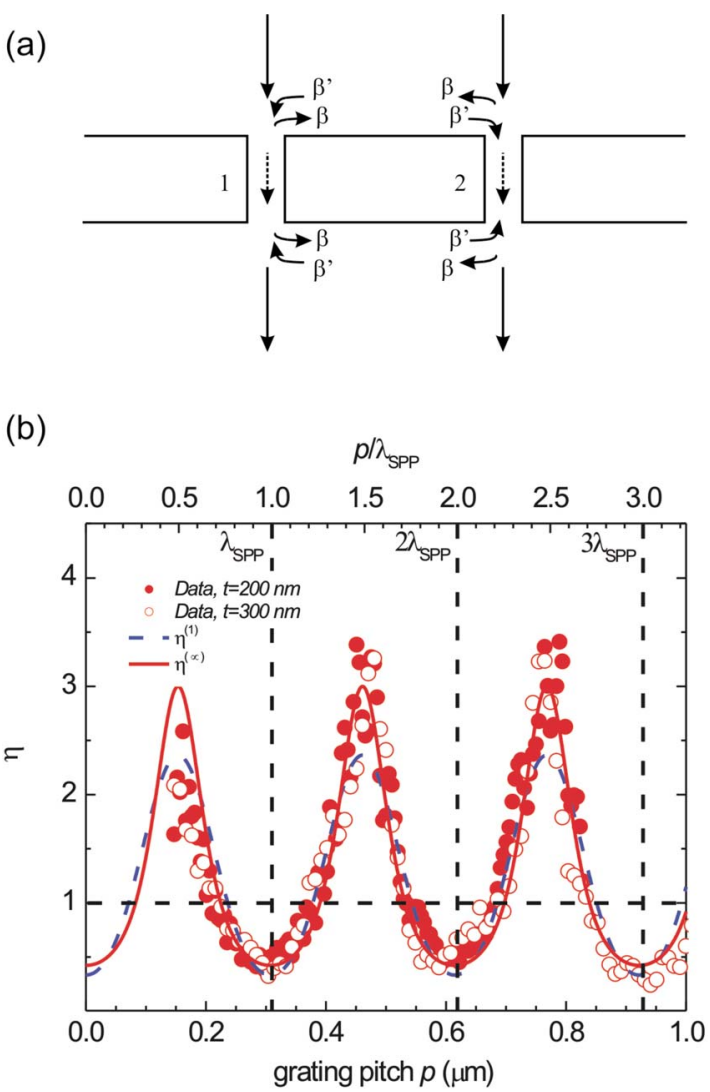

FIG. 4. (Color online) Panel (a) shows a schematic of the interference model used to fit the two-slit transmission intensity. Panel (b) shows the two-slit transmission intensity normalized to singleslit transmission as a function of slit-slit separation and plotted on a linear scale. The data (filled circles) show the transmission profile for a $200 \mathrm{~nm}$ thick Ag film; open circles show similar data for a $300 \mathrm{~nm}$ thick Ag film. The dashed line shows a fit using the firstorder interference model of Eq. (4) (blue curve) and the solid line shows a fit using the infinite-order model of Eq. (5) (red curve).

$$
\begin{aligned}
H_{N=2}= & \left(H_{0}+\beta \beta^{\prime} e^{i k_{\mathrm{SPP} p}} H_{0}\right) T\left(1+\beta \beta^{\prime} e^{i k_{\mathrm{SPP}} p}\right)=H_{0} T(1 \\
& \left.+\beta \beta^{\prime} e^{i k_{\mathrm{SPP} p}}\right)^{2} .
\end{aligned}
$$

The $H$ field at the output aperture of a single slit is given by

$$
H_{N=1}=H_{0} T .
$$

This interference process yields a net transmission intensity (normalized to that of a single slit) given by

$$
\eta^{(1)}(p)=\left\{1+\left(\beta_{0} \beta_{0}^{\prime}\right)^{2}+2 \beta_{0} \beta_{0}^{\prime} \cos \left[\left(\frac{2 \pi}{\lambda_{\mathrm{SPP}}}\right) p+\varphi\right]\right\}^{2},
$$

where $\beta_{0} \beta_{0}^{\prime}=\left|\beta \beta^{\prime}\right|$, and the phase $\varphi=\arg \left(\beta \beta^{\prime}\right)$ is the phase associated with the SPP $\leftrightarrow$ propagating wave conversion, exclusive of the phase accumulated along the surface, $\left(2 \pi / \lambda_{\text {SPP }}\right) p$. Refining the model by taking into account multiple surface wave reflections at the slits results in the following closed-form expression: 


$$
\eta^{(\infty)}(p)=\left\{1+\left(\beta_{0} \beta_{0}^{\prime}\right)^{2}-2 \beta_{0} \beta_{0}^{\prime} \cos \left[\left(\frac{2 \pi}{\lambda_{\mathrm{SPP}}}\right) p+\varphi\right]\right\}^{-2} .
$$

The analysis and resulting expression is analogous to that obtained for a Fabry-Perot cavity containing propagating modes. Here, the cavity "mirrors" are the slits with an effective reflection coefficient $\beta \beta^{\prime}$ and the modes contained in the cavity are surface plasmon polariton modes. Equation (5) is fit to the experimental results [Fig. 4(b)] by fitting parameters, $\lambda_{\mathrm{SPP}}, \beta_{0} \beta_{0}^{\prime}$, and $\varphi$.

\section{B. Comparison of model and measurements}

A fit of $\eta^{\infty}(p)$ to the combined set of experimental transmission data for both $t=300 \mathrm{~nm}$ and $t=200 \mathrm{~nm}$ is shown in Fig. 4(b) (solid red curve). Very good agreement is obtained using fitting parameters $\lambda_{\mathrm{SPP}}=307 \pm 2 \mathrm{~nm}, \quad \beta_{0} \beta_{0}^{\prime}$ $=0.23 \pm 0.01$, and $\varphi=\pi \pm 0.03$. The best-fit value for $\lambda_{\text {SPP }}$ agrees with $\lambda_{\mathrm{SPP}}=309 \mathrm{~nm}$, calculated from Eq. (1), within experimental uncertainty. The shape of $\eta^{\infty}(p)$ is reminiscent of the transmission characteristics of a "lossy" two-mirror Fabry-Perot resonator of free spectral range, $\Delta \lambda=\lambda_{\text {SPP }}$, and with full width at half maximum, $\partial \lambda=0.29 \lambda_{\mathrm{SPP}}$. The "finesse" of the surface wave cavity is then defined by $F$ $=\Delta \lambda / \partial \lambda=3.4$. The Fabry-Perot profile suggests the influence of multiple surface wave reflections at the slit sites but with rather low reflectivity. It is important to emphasize that positions of minima and maxima between a conventional Fabry-Perot cavity and the surface wave cavity are reversed, and this reversal is due to the $\pi$ phase shift between the surface waves and the incident wave. A plot of the first-order model $\eta^{(1)}(p)$ is also included in Fig. 4(b) (dashed blue curve) using the fitting parameters above. The essential profile of the normalized transmission as a function of $p$ is already well reproduced by $\eta^{(1)}(p)$. The first-order fit suggests that the formation of transmission minima is predominantly controlled by interference at the slit openings rather than by the presence of higher order multiple reflections. Assuming that the amplitudes $\beta_{0}, \beta_{0}^{\prime}$ conversion efficiencies are equal, the best-fit value of their product implies that slits convert incident light to surface waves with an efficiency of almost $50 \%$. The positioning of the minima at $p=n \lambda_{\mathrm{SPP}}$ ( $n$ $=1,2, \ldots)$ is due to $\varphi=\pi$ and is in agreement with the find- ings of Ref. 15. It is also consistent with recent calculations showing that diffracted propagating and evanescent surface modes are $\pi$ out of phase. ${ }^{23}$ A simple physical explanation for this phase shift involving surface currents, induced by the standing wave $H$ field at the surface, charging the slits, has recently been proposed. ${ }^{24}$

\section{SUMMARY AND CONCLUSIONS}

In summary, we have measured the transmitted far-field intensity through a series of subwavelength slit arrays as a function of array pitch and have determined that the minimum per-slit transmission at the array output facet occurs for an array pitch equal to an integer number of wavelengths of the surface plasmon polariton. We have also determined that the per-slit transmitted intensity does not increase appreciably above an array size greater than $N=3$. These findings support the view that the transmission profile is controlled by two sequential processes: interference between the incident propagating mode and the principal evanescent surface mode (SPP) on the input side of the film, followed by interference between the emerging light and the SPP on the exit side of the film. Furthermore, at least in the case of slit arrays, the surface wave interaction is essentially confined to adjacent structures rather than characterized by excitation of collective Bloch modes delocalized over the entire array. Finally, in contrast to the minima at pitches equal to integer multiples of $\lambda_{\mathrm{SPP}}$, we find no evidence of regular recurring features (maxima or minima) at array pitches equal to integer multiples of the propagating wavelengths at $\lambda_{0}$ or $\lambda_{1}$.

\section{ACKNOWLEDGMENTS}

Support from the Caltech Kavli Nanoscience Institute, the National Science Foundation under Grant No. DMR 0606472 and the use of facilities at the Center for Science and Engineering of Materials, an NSF Materials Research Science and Engineering Center at Caltech, are gratefully acknowledged. Support from the Ministère délégué à l'Enseignement Supérieur et à la Recherche under the programme ACI-"Nanosciences-Nanotechnologies," the Région Midi-Pyrénées (SFC/CR 02/22), and FASTNet (HPRN-CT2002-00304) EU Research Training Network, as well as from the research foundation FAPESP of the State of São Paulo, Brazil is also gratefully acknowledged.

\footnotetext{
*Present address: Center for Nanoscale Science and Technology, National Institute of Standards and Technology, 100 Bureau Drive, Stop 6203, Gaithersburg, MD 20899-8412.

†jweiner@irsamc.ups-tlse.fr

${ }^{1}$ T. W. Ebbesen, H. J. Lezec, H. F. Ghaemi, T. Thio, and P. A. Wolf, Nature (London) 391, 667 (1998).

${ }^{2}$ E. Popov, M. Nevière, S. Enoch, and R. Reinisch, Phys. Rev. B 62, 16100 (2000).

${ }^{3}$ L. Martin-Moreno, F. J. Garcia-Vidal, H. J. Lezec, K. M. Pellerin, T. Thio, J. B. Pendry, and T. W. Ebbesen, Phys. Rev. Lett. 86,
}

1114 (2001).

${ }^{4}$ M. Sarrazin, J.-P. Vigneron, and J.-M. Vigoureux, Phys. Rev. B 67, 085415 (2003).

${ }^{5}$ C. Genet, M. P. van Exter, and J. P. Woerdman, Opt. Commun. 225, 331 (2003).

${ }^{6}$ S.-H. Chang, S. K. Gray, and G. C. Schatz, Opt. Express 13, 3150 (2005).

${ }^{7}$ F. J. Garcia de Abajo, J. J. Sáenz, I. Campillo, and J. S. Dolado, Opt. Express 14, 7 (2006).

${ }^{8}$ J. A. Porto, F. J. Garcia-Vidal, and J. B. Pendry, Phys. Rev. Lett. 
83, 2845 (1999).

${ }^{9}$ Ph. Lalanne, J. P. Hugonin, S. Astilean, M. Palamaru, and K. D. Möller, J. Opt. A, Pure Appl. Opt. 2, 48 (2000).

${ }^{10}$ Y. Takakura, Phys. Rev. Lett. 86, 5601 (2001).

${ }^{11}$ Q. Cao and Ph. Lalanne, Phys. Rev. Lett. 88, 057403 (2002).

${ }^{12}$ P. Lalanne, C. Sauvan, J. P. Hugonin, J. C. Rodier, and P. Chavel, Phys. Rev. B 68, 125404 (2003).

${ }^{13}$ Y. Xie, A. R. Zakharian, J. V. Moloney, and M. Mansuripur, Opt. Express 13, 4485 (2005).

${ }^{14}$ V. Mikhailov, G. A. Wurtz, J. Elliott, P. Bayvel, and A. V. Zayats, Phys. Rev. Lett. 99, 083901 (2007).

${ }^{15}$ O. T. A. Janssen, H. P. Urbach, and G. W. 't Hooft, Opt. Express 14, 11823 (2006).

${ }^{16}$ B. Ung and Y. Sheng, Opt. Express 15, 1182 (2007).
${ }^{17}$ H. J. Lezec and T. Thio, Opt. Express 12, 3629 (2004).

${ }^{18}$ G. Gay, O. Alloschery, B. Viaris de Lesegno, J. Weiner, and H. J. Lezec, Phys. Rev. Lett. 96, 213901 (2006).

${ }^{19}$ G. Gay, O. Alloschery, B. Viaris de Lesegno, C. O’Dwyer, J. Weiner, and H. J. Lezec, Nat. Phys. 2, 262 (2006).

${ }^{20}$ F. Kalkum, G. Gay, O. Alloschery, J. Weiner, H. J. Lezec, Y. Xie, and M. Mansuripur, Opt. Express 15, 2613 (2007).

${ }^{21}$ Y. Xie, A. R. Zakharian, J. V. Moloney, and M. Mansuripur, Opt. Express 14, 6400 (2006).

${ }^{22}$ H. Raether, Surface Plasmons on Smooth and Rough Surfaces and on Gratings, (Springer-Verlag, Berlin, 1988).

${ }^{23}$ G. Lévêque, O. J. F. Martin, and J. Weiner, Phys. Rev. B 76, 155418 (2007).

${ }^{24}$ J. Weiner, Opt. Express 16, 950 (2008). 